\title{
Effects of spontaneous otoacoustic emissions on frequency discrimination
}

\author{
Hansen, Rói; Santurette, Sébastien; Verhulst, Sarah
}

Published in:

Meetings on Acoustics. Proceedings

Link to article, DOI:

$10.1121 / 1.4799663$

Publication date:

2013

Document Version

Publisher's PDF, also known as Version of record

Link back to DTU Orbit

Citation (APA):

Hansen, R., Santurette, S., \& Verhulst, S. (2013). Effects of spontaneous otoacoustic emissions on frequency discrimination. Meetings on Acoustics. Proceedings, 19. https://doi.org/10.1121/1.4799663

\section{General rights}

Copyright and moral rights for the publications made accessible in the public portal are retained by the authors and/or other copyright owners and it is a condition of accessing publications that users recognise and abide by the legal requirements associated with these rights.

- Users may download and print one copy of any publication from the public portal for the purpose of private study or research.

- You may not further distribute the material or use it for any profit-making activity or commercial gain

- You may freely distribute the URL identifying the publication in the public portal

If you believe that this document breaches copyright please contact us providing details, and we will remove access to the work immediately and investigate your claim 


\section{Proceedings of Meetings on Acoustics}
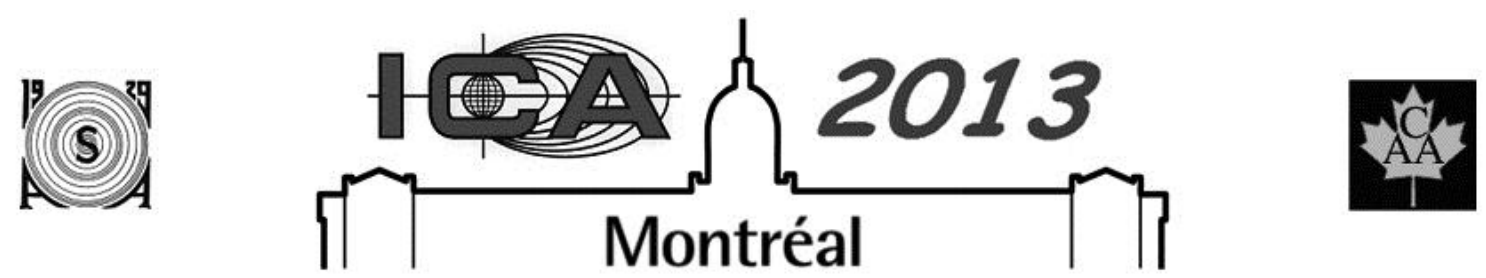

ICA 2013 Montreal

Montreal, Canada

2 - 7 June 2013

\section{Psychological and Physiological Acoustics}

\section{Session 3aPP: Auditory Physiology and Modeling (Poster Session)}

\section{3aPP26. Effects of spontaneous otoacoustic emissions on frequency discrimination}

Rói Hansen, Sébastien Santurette* and Sarah Verhulst

*Corresponding author's address: Centre for Applied Hearing Research, Technical University of Denmark, Ørsteds Plads 352, Kgs. Lyngby, 2800, None, Denmark, ses@elektro.dtu.dk

When an external tone is presented in proximity to the frequency of a spontaneous otoacoustic emission (SOAE), the SOAE typically synchronizes to the external tone, a phenomenon known as "entrainment". As the tone moves further away from the SOAE frequency, beating patterns between the SOAE and the pure tone occur (Long, Hear. Res. 119, 1998). This study investigated perceptual consequences of SOAE beating and entrainment on the frequency difference limen (FDL), which has been found to improve near an SOAE. SOAE entrainment patterns were obtained for six subjects with a strong SOAE in the ipsilateral ear and no SOAE in the corresponding frequency range of the contralateral ear. Hearing thresholds and FDLs were measured ipsi- and contralaterally for nine frequencies covering the entrainment and beating regions of the SOAE. FDLs systematically improved in the entrainment region, worsened when beating occurred, and improved again for frequencies further away from the SOAE. No improvement in FDL was found in any of the contralateral ears tested, suggesting that the effect is of peripheral, rather than of central, origin. The results contradict an earlier hypothesis stating that FDL performance near SOAE frequencies is governed by a central oversensitivity to the SOAE frequency.

Published by the Acoustical Society of America through the American Institute of Physics 


\section{INTRODUCTION}

The existence of sounds originating in the inner ear, known as otoacoustic emissions (OAEs), was predicted by Gold (1948) based on the argument that the cochlea was not simply a passive system, but would spontaneously produce sounds due to active feedback mechanisms, a prediction later confirmed by Kemp (1978). OAEs can be recorded in at least one third of human ears in the absence of external acoustic stimulation (Probst et al., 1991). Such spontaneous OAEs (SOAEs) are narrow-band, pure-tone-like sounds, thought to be generated by low-level spontaneous hair-cell activity producing a standing wave in the cochlea, and are transmitted via the ossicular chain and the tympanic membrane towards the ear canal, where they can be recorded (Kemp, 2008). Although SOAEs are not known to serve any functional purpose, there is evidence that they affect the perception of external sounds.

Specifically, Köhler and Fritze (1994) found that the perceived pitch of pure tones close in frequency to an SOAE was shifted in the emission ear compared to the pitch of the same pure tones presented in the opposite ear containing no SOAE, with upward and downward pitch shifts above and below the SOAE frequency, respectively. The same authors reported having observed poorer frequency discrimination at and around the SOAE frequency than in frequency regions without SOAE. In contrast, Norena et al. (2002) found that pure-tone frequency discrimination systematically improved near an SOAE frequency, such that reduced frequency difference limens (FDLs) were observed for monaural stimulation of the emission ear, but also of the contralateral ear containing no SOAE. Due to these similar variations in FDL ipsilaterally and contralaterally to the emission ear, Norena et al. (2002) argued that the observed effect was of a central origin, possibly due to prolonged ongoing stimulation of auditory nerve cells tuned to the SOAE frequency throughout life, which might lead to an overrepresentation of that frequency at a cortical level and thus facilitate discrimination.

In addition to this suggestion of a central plasticity effect, there is strong evidence that the peripheral interaction of SOAEs with external tones is consistent with changes in the perceived quality of pure tones close in frequency to an SOAE (e.g., Zurek,1981; Long,1998). Long and Tubis (1988) and Long (1998) recorded ear-canal sound pressure fluctuations as a pure tone was swept past an SOAE and observed level-dependent beating patterns between the SOAE and the external tone. While clear level fluctuations were observed when the tone frequency was sufficiently remote from the SOAE frequency, this beating disappeared as the tone approached the SOAE, indicating a synchronization of the emission to the tone frequency, a phenomenon known as "entrainment". Such entrainment patterns were consistent with near-threshold pure tones being perceived as "cricket-like" or "rough" in the region of beating with the SOAE, while this roughness sensation disappeared and the stimulus quality was tone-like again in the entrainment region (Fig. 6 in Long and Tubis, 1988).

In the present study, it was hypothesized that this correspondence between the peripheral interaction of SOAEs with external tones and the perceived tonal quality of such tones may be responsible for the variations in FDLs observed in the vicinity of SOAEs. If so, the influence of SOAEs on frequency discrimination may be partly of peripheral origin and not reflect purely central mechanisms, as argued by Norena et al. (2002). The aims were to (1) clarify the conflicting literature reports of elevated $v s$ reduced FDLs around SOAEs, (2) confirm the presence of FDL variations in both the ipsilateral and the contralateral ear, and (3) investigate whether FDLs are consistent with the beating and entrainment patterns measured at a similar sensation level (SL) in individual subjects.

Using a method similar to that of Long (1998), SOAE entrainment patterns were measured in subjects with a clearly identifiable SOAE in one ear and no SOAE in the same frequency range in the opposite ear. Individual frequency ranges for entrainment and beating for a swept tone at approximately $10 \mathrm{~dB}$ SL were then determined, and 9 test frequencies chosen such that they covered the spectral ranges of entrainment and beating at different rates. Absolute hearing thresholds (HTs) and FDLs for pure tones at $10 \mathrm{~dB}$ SL were then determined at all test frequencies in the two ears of the same subjects. If frequency-discrimination performance follows the changes in tonal quality observed by Long and Tubis (1988), higher FDLs would be expected in regions of perceivable beating than in the entrainment region, and FDLs may decrease again for tone frequencies remote from the SOAE. The details of the experimental methods are presented below, followed by the objective and subjective results and a discussion of these. In the following, the terms "ipsilateral" and "contralateral" always refer to the ear with the SOAE of interest and to the opposite non-emission ear, respectively. 


\section{METHODS}

\section{Subjects and Experimental Setup}

Thirty subjects were screened for the presence of SOAEs. Six subjects with no reported history of hearing difficulties (3 females, ages 24-30 yrs) who had SOAE amplitudes larger than $15 \mathrm{~dB}$ above the noise floor and no SOAE within a $\pm 100-\mathrm{Hz}$ range in the contralateral ear were selected. All measurements took place inside an IEC soundproof and electromagnetically shielded booth. The subjects were seated in a comfortable leaning chair which enabled support for the head to reduce noise from movement, and were instructed to remain as quiet as possible during the measurements. A PC with MATLAB software served as the main platform, with an ASIO sound driver and an RME Fireface 800 soundcard for the physical input and output. The RME output was fed into a 2-channel Etymotic Research ER-10B+ low-noise microphone system via a Tucker Davis Technologies HB7 headphone driver. For the OAE measurements, the recorded signal from the ER-10B+ microphone was preamplified $(+40 \mathrm{~dB})$ then passed through a Rockland Model 852 bandpass filter $(0.6-5 \mathrm{kHz})$ and fed into the RME input. The sampling frequency was $48 \mathrm{kHz}$. The system was calibrated using a B\&K 4157 ear simulator with a B\&K 2669 Falcon Range $1 / 2$ " microphone preamplifier, a B\&K 2636 sound-level meter, and a B\&K 4230 calibrator. The measurement and analysis methods described below were implemented in MATLAB using the pawavplay software for playback and recording. The same equipment was used for objective and behavioral measurements. For the latter, the listeners reported their answers via a computer keyboard and were provided visual feedback via a computer screen. The monitor was turned off and disconnected from the system during all objective measurements to prevent electromagnetic interference. All measurements were performed in two sessions on separate days, one for all ipsilateral measurements, and one for all contralateral measurements. For each subject, the measurements took place in the same order as described below. The duration of the ipsi- and contralateral sessions was about 4.5 and 2.5 hours, respectively. As SOAEs are known to drift in level and frequency up to $30 \mathrm{~min}$ after a probe is inserted into or removed from the ear canal (Whitehead, 1991), the subjects kept the ER-10B+ probe or an identical dummy probe inside the ipsilateral ear canal throughout the first measurement session, also during breaks.

\section{SOAE Frequency and Level}

The SOAE spectrum was first measured in both ears. Prior to this, a 120-s long control recording in the presence of a $1-\mathrm{kHz}$ pure-tone at $15 \mathrm{~dB}$ SPL was performed to ensure that the levels determined from the measurements were accurate. Subsequently, 180 -s recordings were obtained without stimulation to obtain the emission spectra. The ipsiand contralateral spectra were analyzed and the SOAE center frequency, $\mathrm{F}_{0}$, and level, $\mathrm{L}_{0}$, determined by applying a power spectrum estimation (PSE) procedure to the recorded ear-canal sound pressure. The PSE method used here was based on the Welch method and similar to that used by Talmadge et al. (1993). The discrete time domain signal was first divided into segments of 1 -s duration and $50 \%$ overlap. Artifact rejection was then performed to improve the signal-to-noise ratio by ranking segments with respect to peak-amplitude excursion and discarding a percentage of the highest ranking segments. The subject-dependent percentage of discarded segments varied from $10-40 \%$ and was selected such that no peak excursions were observable in the remaining segments. Windowing followed by a fast Fourier transform were then applied to each segment. The segments were then added and rescaled with a factor inversely proportional to the power of the window, to compensate for changes in amplitude due to windowing. The SOAE level $\mathrm{L}_{0}$ was defined as the spectral peak-amplitude of the SOAE.

\section{Entrainment Patterns}

The variations in temporal pressure were recorded in the ipsilateral ear canal while presenting a 60 -s ascending sweep around $\mathrm{F}_{0}( \pm 50 \mathrm{~Hz})$. The measured sound pressure was bandpass filtered with a center frequency of $\mathrm{F}_{0}$ and a subject-dependent bandwidth between 30 and $60 \mathrm{~Hz}$, chosen such that the entrainment and beating regions were visually identifiable. Fullwave rectification was then applied and the temporal envelope was extracted using lowpass filtering (20-Hz cutoff). This resulted in a pattern of pressure fluctuations as a function of time, i.e., as the instantaneous frequency of the swept tone is increased. The procedure was repeated for sweep levels between -10 $\mathrm{dB}$ and $+15 \mathrm{~dB}$ relative to $\mathrm{L}_{0}$, in steps of $5 \mathrm{~dB}$. The range of sweep levels generating detectable temporal variations was subject dependent, such that between 4 and 6 of these 5-dB steps were measured in each subject.

After the temporal entrainment patterns were obtained, the SOAE measurement in the ipsilateral ear was repeated in order to check whether the emission had drifted in frequency and level due to the presence of the probe 
in the ear canal (Whitehead, 1991). If so, $\mathrm{F}_{0}$ and $\mathrm{L}_{0}$ were adjusted for use in all following measurements, and the SOAE frequency and level were assumed to be stable from that point.

\section{Hearing Thresholds}

Absolute hearing thresholds (HTs) were measured in the ipsilateral ear using the adaptive single-interval updown method (Lecluyse and Meddis, 2009). In this procedure, a single interval containing two pure tones at the tested frequency is presented to the subject, the second tone always being $10 \mathrm{~dB}$ lower in level than the first tone. The task of the subject is to indicate the number of tones heard in each trial, after which the level of the two tones is varied adaptively. The 500-ms tones were separated by a 500-ms silent gap and gated with 50-ms on- and offset ramps. Stepsizes of 10,6 , and $2 \mathrm{~dB}$ were used with a total of 7 reversals, providing a threshold accuracy of $2 \mathrm{~dB}$ (Lecluyse and Meddis, 2009). Two repetitions of the procedure were performed for each test frequency, and the final HT was defined as the average threshold over these two repetitions.

\section{Entrainment and Beating Regions}

For a given subject, the HT at $\mathrm{F}_{0}$ was determined first, and the entrainment pattern measured for the closest sweep level to $10 \mathrm{~dB} \mathrm{SL}$ at $\mathrm{F}_{0}$ was identified. The spectral widths of the entrainment regions below and above $\mathrm{F}_{0}$, $\Delta \mathrm{F}_{\mathrm{E}-}$ and $\Delta \mathrm{F}_{\mathrm{E}+}$, were then determined graphically by estimating the frequencies of transition between full entrainment and intermittent beating. The remaining test frequencies $\mathrm{F}_{-4}, \mathrm{~F}_{-3}, \mathrm{~F}_{-2}, \mathrm{~F}_{-1}, \mathrm{~F}_{1}, \mathrm{~F}_{2}, \mathrm{~F}_{3}$, and $\mathrm{F}_{4}$, were calculated by subtracting and adding fixed proportions $\mathrm{p}_{x}$ of $\Delta \mathrm{F}_{\mathrm{E}-\text { and }} \Delta \mathrm{F}_{\mathrm{E}+}$, respectively, to $\mathrm{F}_{0}$, such that $\mathrm{F}_{x}=\mathrm{F}_{0}-$ $\mathrm{p}_{x} \Delta \mathrm{F}_{\mathrm{E}-}$ for $x<0$ and $\mathrm{F}_{x}=\mathrm{F}_{0}+\mathrm{p}_{x} \Delta \mathrm{F}_{\mathrm{E}+}$ for $x>0$. These proportions $\mathrm{p}_{x}$ were set to $0.75,3.5,7$, and 14 for $|x|$ equal to 1 , 2, 3, and 4, respectively, such that $F_{-1}, F_{0}$, and $F_{1}$ fell in the entrainment region, while perceivable beating was expected to occur at $\mathrm{F}_{-3}, \mathrm{~F}_{-2}, \mathrm{~F}_{2}$, and $\mathrm{F}_{3}$, and reduced interaction with the SOAE occurred at $\mathrm{F}_{-4}$ and $\mathrm{F}_{4}$. Once the test frequencies were determined, HTs were measured at all 9 frequencies with the method described above.

The presence of entrainment at each test frequency $F_{x}$ was then also determined spectrally by repeating the SOAE measurement for $120 \mathrm{~s}$ in the presence of a $10-\mathrm{dB}-\mathrm{SL}$ pure-tone stimulus at $\mathrm{F}_{x}$. If the level or frequency of the SOAE was affected by the presence of the external tone, this would confirm that the emission was entrained or partially entrained. In the case of complete entrainment, the SOAE would not be distinguishable from the external tone in the measured spectrum. If the SOAE remained unaffected, this would indicate that $\mathrm{F}_{x}$ lies outside the entrainment region. This power-spectrum verification was performed with pure tones at each test frequency $\mathrm{F}_{x}$. The SOAE was also remeasured (without stimulation) to verify that it had remained stable in level and frequency.

\section{Frequency Difference Limens}

FDLs were measured as a percentage of $\mathrm{F}_{x}$ with a two-interval, two-alternative, forced-choice paradigm and a one-up, two-down procedure tracking the $70.7 \%$ correct point of the psychometric function (Levitt, 1971). In each trial, two 500-ms tones with frequencies $\mathrm{F}_{\text {low }}$ and $\mathrm{F}_{\text {high }}$, logarithmically equidistant from $\mathrm{F}_{x}$, were presented, such that

$$
\left\{\begin{array}{l}
\mathrm{F}_{\text {low }}=\frac{\mathrm{F}_{x}}{2}\left(\sqrt{4+\alpha^{2}}-\alpha\right) \\
\mathrm{F}_{\text {high }}=\frac{\mathrm{F}_{x}}{2}\left(\sqrt{4+\alpha^{2}}+\alpha\right), \text { where } \alpha=\left(\mathrm{F}_{\text {high }}-\mathrm{F}_{\text {low }}\right) / \mathrm{F}_{x} \text { is the tracking variable. }
\end{array}\right.
$$

The presentation order of the two tones, separated by a 250-ms silent gap and gated with 20-ms onset and offset ramps, was random. The task of the subject was to identify which of the first or second tone was higher in frequency. Responses were given by pressing the " 1 " or " 2 " key on a computer keyboard, and visual feedback was provided following each trial. In each measurement block, the starting value of $\alpha$ was $4 \%$ and $\alpha$ was initially divided by a factor of 4 after two consecutive correct responses, and multiplied by the same factor after each incorrect response. This factor was reduced to 2 and $\sqrt{ } 2$ after the second and the fourth reversal, respectively, after which 6 reversals with this final stepsize were obtained. The threshold was defined as the geometric mean of all points over these last 6 reversals. The absolute presentation levels of $F_{\text {low }}$ and $F_{\text {high }}$ were frequency-dependent such that they always corresponded to $10 \mathrm{~dB}$ SL according to a linear interpolation of the measured HTs between the 9 test 
frequencies $\mathrm{F}_{x}$. Each listener performed one measurement block for each value of $\mathrm{F}_{x}$ and repeated the experiment 4 times. The final FDL for each subject was determined by averaging the last 3 repetitions.

\title{
Contralateral Measurements
}

The contralateral measurements were carried out in a second experimental session. As for the ipsilateral conditions, the emission spectrum was remeasured in both ears and the frequency and amplitude of the SOAE determined, to check whether $\mathrm{F}_{0}$ and $\mathrm{L}_{0}$ had shifted compared to the first session. HTs and FDLs at the same test frequencies $\mathrm{F}_{x}$ as in the ipsilateral ear were then measured with the same procedure as described above.

\section{RESULTS}

\section{Objective Results: SOAE Characteristics}

\author{
SOAE Frequency and Level
}

The frequencies $\mathrm{F}_{0}$ and levels $\mathrm{L}_{0}$ of the SOAEs of interest for each subject are given in Table 1. The values measured at the beginning of the experimental session are indicated in brackets in addition to the stabilized values obtained about 30 min after probe insertion. A slight drift in frequency and/or level was observed in most subjects, typically downwards in frequency, as reported by Whitehead (1991). Figure 1(a) shows the ipsilateral (black curve) and contralateral (green curve) SOAE spectra for one example subject (S1). The bottom panel shows a close-up of these two spectra around $\mathrm{F}_{0}$ and the ipsilateral spectrum measured $30 \mathrm{~min}$ after probe insertion (red dashed curve).

TABLE 1. SOAE frequencies $\left(\mathrm{F}_{0}\right)$ and levels $\left(\mathrm{L}_{0}\right)$ for each of the six subjects, measured about 30 min after probe insertion as well as at the beginning of the experimental session (in brackets). The width of the estimated entrainment region at $10 \mathrm{~dB}$ SL below $\left(\Delta \mathrm{F}_{\mathrm{E}-}\right)$ and above $\left(\Delta \mathrm{F}_{\mathrm{E}+}\right)$ the SOAE frequency are also shown.

\begin{tabular}{lllllll}
\hline & S1 & S2 & S3 & S4 & S5 & S6 \\
\hline $\mathrm{F}_{0}(\mathrm{~Hz})$ & $1241(1242)$ & $4091(4095)$ & $3009(3012)$ & $1440(1439)$ & $1965(1967)$ & $2068(2068)$ \\
$\mathrm{L}_{0}(\mathrm{~dB} \mathrm{SPL})$ & $20.1(19.9)$ & $14.5(14.2)$ & $6.5(6.2)$ & $7.4(8.2)$ & $2.8(3.7)$ & $9.4(7.0)$ \\
\hline$\Delta \mathrm{F}_{\mathrm{E}-}(\mathrm{Hz})$ & 5.5 & 27.0 & 5.8 & 6.2 & 14.0 & 4.5 \\
$\Delta \mathrm{F}_{\mathrm{E}+}(\mathrm{Hz})$ & 3.8 & 35.0 & 7.0 & 5.6 & 8.5 & 4.0 \\
\hline $\mathrm{F}_{-4}(\mathrm{~Hz})$ & 1164 & 3713 & 2896 & 1353 & 1771 & 2005 \\
$\mathrm{~F}_{-3}(\mathrm{~Hz})$ & 1203 & 3902 & 2952 & 1397 & 1869 & 2037 \\
$\mathrm{~F}_{-2}(\mathrm{~Hz})$ & 1222 & 3997 & 2981 & 1418 & 1918 & 2052 \\
$\mathrm{~F}_{-1}(\mathrm{~Hz})$ & 1237 & 4071 & 3003 & 1435 & 1957 & 2065 \\
$\mathrm{~F}_{1}(\mathrm{~Hz})$ & 1244 & 4117 & 3017 & 1444 & 1973 & 2071 \\
$\mathrm{~F}_{2}(\mathrm{~Hz})$ & 1254 & 4214 & 3044 & 1460 & 1997 & 2082 \\
$\mathrm{~F}_{3}(\mathrm{~Hz})$ & 1268 & 4336 & 3079 & 1479 & 2027 & 2096 \\
$\mathrm{~F}_{4}(\mathrm{~Hz})$ & 1294 & 4581 & 3148 & 1518 & 2086 & 2124 \\
\hline
\end{tabular}

\section{Entrainment and Beating Regions}

Figure 1(b) shows the temporal entrainment patterns obtained in subject S1 as an external tone was swept around $\mathrm{F}_{0}$ at different levels relative to $\mathrm{L}_{0}$. As observed by Long and Tubis (1988) and Long (1998), ear-canal pressure fluctuations occurred when the frequency of the external tone was sufficiently remote from $\mathrm{F}_{0}$, while this beating pattern disappeared as the frequency of the external tone approached $\mathrm{F}_{0}$, indicating synchronization of the $\mathrm{SOAE}$ with the external tone. The width of the latter entrainment region was found to increase with presentation level in all subjects, consistent with the observations of Long and Tubis (1988) and Long (1998). After measurement of the subjects' $\mathrm{HT}$ at $\mathrm{F}_{0}$, the entrainment patterns corresponding to $10 \mathrm{~dB}$ SL were selected and the individual widths of the corresponding entrainment regions below $\left(\Delta \mathrm{F}_{\mathrm{E}-}\right)$ and above $\left(\Delta \mathrm{F}_{\mathrm{E}+}\right) \mathrm{F}_{0}$ were estimated. For the example of subject $\mathrm{S} 1$ shown in Fig. 1(b), the measured $\mathrm{HT}$ at $\mathrm{F}_{0}$ was $7.6 \mathrm{~dB} \mathrm{SPL}$, i.e., $12.5 \mathrm{~dB}$ below $\mathrm{L}_{0}$, such that $10 \mathrm{~dB} \mathrm{SL}$ corresponded to a level of $-2.5 \mathrm{~dB}$ relative to $\mathrm{L}_{0}$. The width of the entrainment region was thus determined visually using the $-5 \mathrm{~dB}$ entrainment pattern (Fig. 1(b), red arrows). These estimated values as well as the derived test frequencies $\mathrm{F}_{x}$ are specified in Table 1 for all subjects. The total width of the entrainment region $\Delta \mathrm{F}_{\mathrm{E}_{-}}+\Delta \mathrm{F}_{\mathrm{E}+}$ ranged from $0.4 \%$ to $1.5 \%$ of $\mathrm{F}_{0}$, with a general trend of increasing width with $\mathrm{F}_{0}$. 
(a)
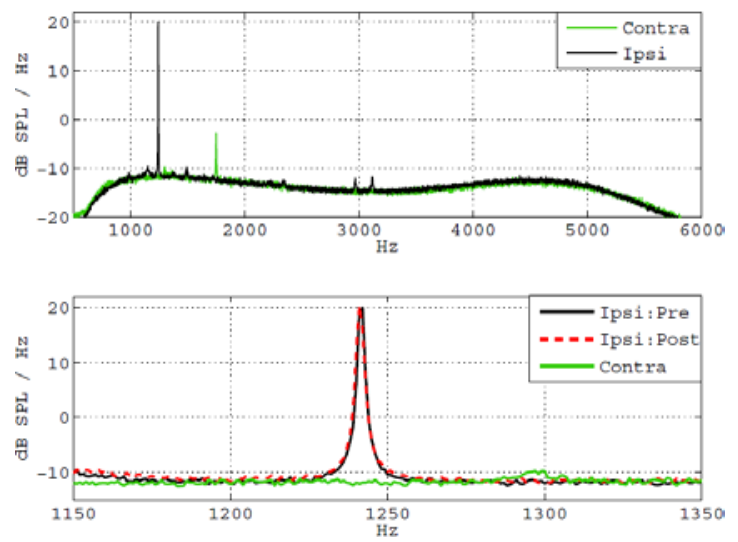

(b)

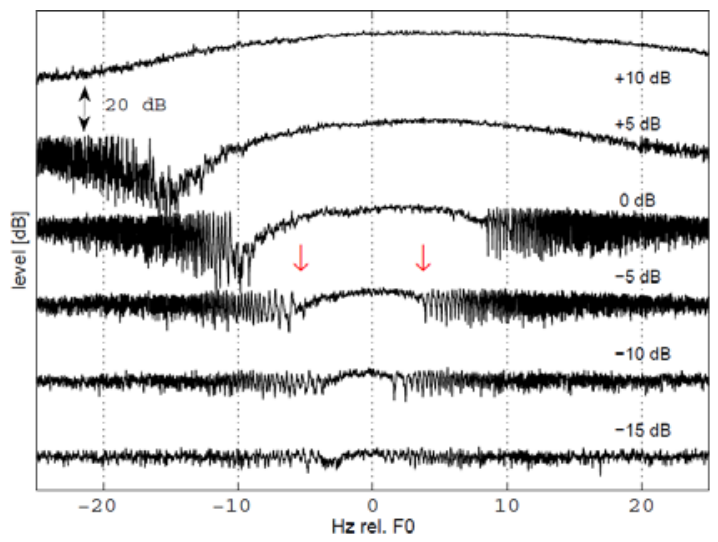

FIGURE 1. (a) Top panel: Broadband SOAE spectra in the ipsilateral (Ipsi, black curve) and contralateral (Contra, green curve) ear of subject S1. Bottom panel: Close-ups on the spectra of the SOAEs of interest for the same subject, measured in the ipsilateral ear at the beginning of the experimental session (Ipsi:Pre, black solid curve) and 30 minutes later (Ipsi:Post, red dashed curve), as well as in the contralateral ear (Contra, solid green curve). (b) SOAE entrainment patterns measured in the same subject with linear sweeps in level steps of $5 \mathrm{~dB}$ relative to the SOAE level. Patterns are displaced by $20 \mathrm{~dB}$ on the ordinate axis for better visualization. The red arrows indicate the estimated boundaries of the entrainment region for the pattern measured at a level closest to $10 \mathrm{~dB}$ SL.

The SOAE levels measured in the presence of 10-dB-SL external tones at $\mathrm{F}_{x}$ are shown in Fig. 2 for each individual subject (top panels, crosses). The presence of entrainment, reflected by a drop in level of the SOAE compared to absent stimulation (triangles), was confirmed at $\mathrm{F}_{-1}$ and $\mathrm{F}_{1}$ in all subjects. Although the $\mathrm{F}_{x}$ values were chosen such that $\mathrm{F}_{x}$ would fall outside the entrainment region for $|\mathrm{x}| \geq 2$, substantial reductions in SOAE level were also observed at $\mathrm{F}_{2}$ for subjects $\mathrm{S} 1$ and $\mathrm{S} 4$, and up to $|\mathrm{x}|=3$ for subject $\mathrm{S} 2$, indicating a wider spectral range of entrainment than expected in these subjects.

\section{Subjective Results: Absolute Thresholds and Frequency Difference Limens}

\section{Absolute Hearing Thresholds}

The mean individual HTs are shown in Fig. 2 (top panels, circles) for the ipsilateral (solid black curves) and contralateral (dashed red curve) ear. Two-way ANOVA statistics revealed significant effects of $\mathrm{F}_{x}[F(8,40)=3.83$, $p=0.002]$ and subject $[F(5,40)=7.14, p=0.0001]$ on HTs in the ipsilateral ear, while there was no effect of $\mathrm{F}_{x}$ $[F(8,40)=0.68, p=0.704]$ and a significant effect of subject $[F(5,40)=0.68, p<0.0001]$ in the contralateral ear. Post hoc multiple comparisons using Tukey's honestly significant difference criterion indicated that ipsilateral HTs only differed significantly between $\mathrm{F}_{0}$ and $\mathrm{F}_{-3}$ [95\% CI: 1.72-11.54] and between $\mathrm{F}_{0}$ and $\mathrm{F}_{4}$ [95\% CI: 1.07-10.89]. This is consistent with the lower absolute thresholds typically found around SOAE frequencies (e.g., Schloth, 1983).

\section{Frequency Difference Limens}

The individual FDLs are shown in Fig. 2 (bottom panels) for the ipsilateral (solid black curves) and contralateral (dashed red curve) ear. For each subject, the geometric mean and standard deviation over the last 3 measurement blocks are shown. In all subjects, the ipsilateral FDLs were lowest around the SOAE frequency and increased at frequencies further away from $\mathrm{F}_{0}$. These individual patterns were largely consistent with the presence of entrainment estimated from the level drift of the SOAE in the presence of external tones, with lower FDLs in regions of complete entrainment and elevated FDLs when the entrainment was partial or absent (compare FDLs in bottom panel to crosses in top panel for each subject). In contrast to the "U" or "V" shape of the FDL results observed ipsilaterally, the contralateral FDLs did not show a clear dependence on $\mathrm{F}_{x}$ in any of the subjects.

Figure 3 shows the mean FDLs over all subjects, measured ipsilaterally (solid black curve) and contralaterally (dashed red curve) to the SOAE at each of the 9 test frequencies $\mathrm{F}_{x}$. For the ipsilateral conditions, a repeatedmeasures two-way ANOVA on the log-transformed FDLs revealed significant main effects of $\mathrm{F}_{x}[F(8,108)=26.59$, $p<0.0001]$ and subject $[F(5,108)=7.52, p<0.0001]$, as well as a significant interaction between $\mathrm{F}_{x}$ and subject 
$[F(40,108)=3.68, p<0.0001]$. For the contralateral conditions, the same analysis showed no significant main effects of $\mathrm{F}_{x}[F(8,108)=1.94, p=0.061]$ or subject $[F(5,108)=1.74, p=0.131]$. Post hoc multiple comparisons using Tukey's honestly significant difference criterion confirmed that none of the contralateral FDLs differed significantly from one another. However, the same comparisons between ipsilateral FDLs obtained at the SOAE frequency (F0) and in the regions of strongest beating $\left(\mathrm{F}_{-3}, \mathrm{~F}_{-2}, \mathrm{~F}_{2}\right.$, and $\left.\mathrm{F}_{3}\right)$ indicated that the FDLs were significantly elevated at all of these frequencies compared to $\mathrm{F}_{0}\left[95 \%\right.$ CIs: 0.59-1.09 $\left.\left(\mathrm{F}_{-3}\right) ; 0.52-1.04\left(\mathrm{~F}_{-2}\right) ; 0.28-0.80\left(\mathrm{~F}_{2}\right) ; 0.58-1.10\left(\mathrm{~F}_{3}\right)\right]$.

A comparison of ipsilateral and contralateral FDLs using uncorrected double-tailed paired $t$-tests indicated that these differed significantly at $\mathrm{F}_{-2}(p=0.008), \mathrm{F}_{0}(p=0.036)$, and $\mathrm{F}_{1}(p=0.047)$. Finally, the mean log-transformed FDLs were not found to significantly correlate with mean HTs (Spearman's $\rho=0.22, p=0.104$ ).

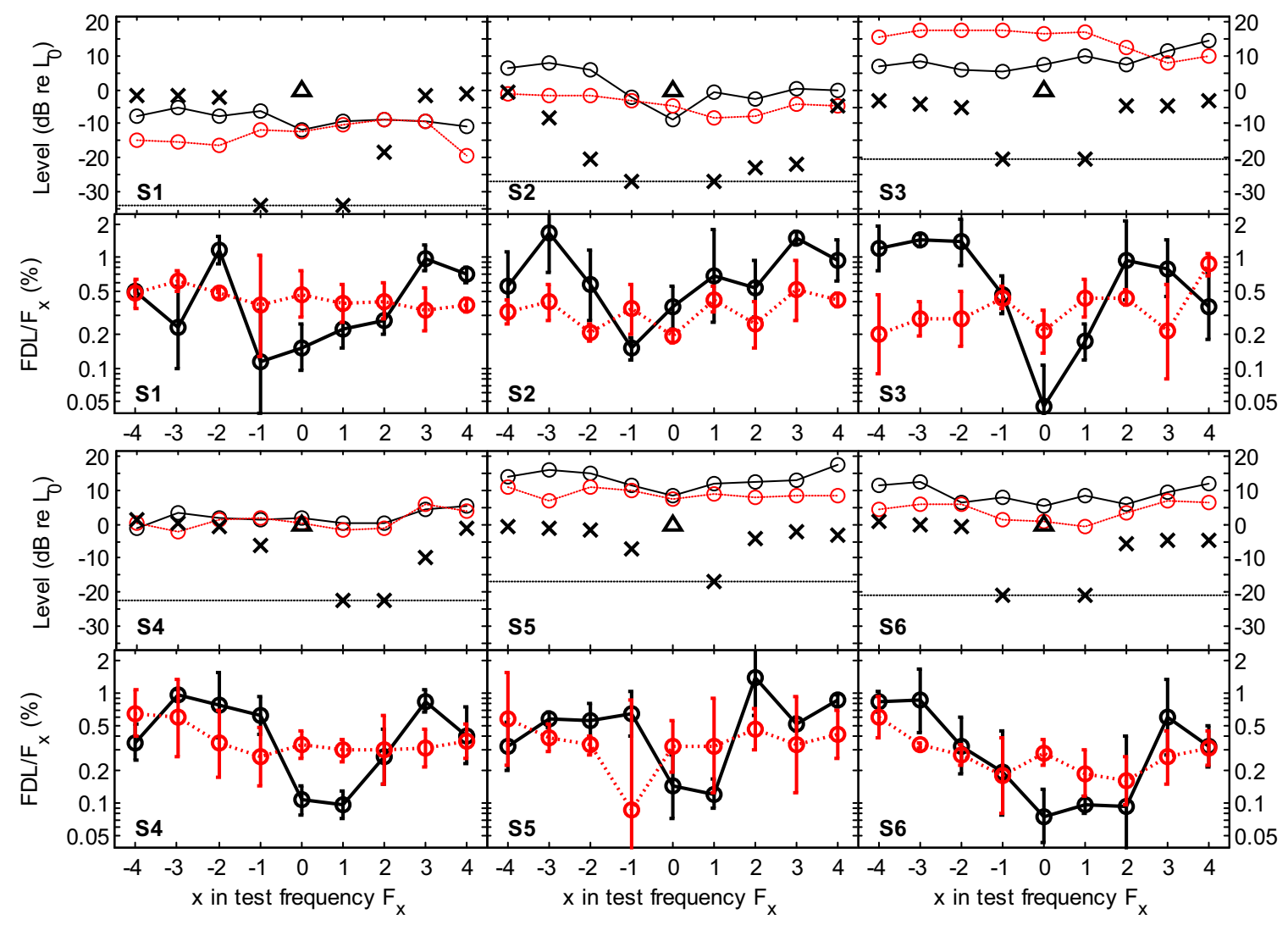

FIGURE 2. Individual objective and subjective results as a function of test frequency $\mathrm{F}_{x}$. Top panels: For each subject, the puretone hearing thresholds measured ipsilaterally (solid black curve) and contralaterally (dashed red curve) to the emission ear are shown relative to the level of the SOAE of interest $\mathrm{L}_{0}$, indicated by the triangle. Crosses indicate the SOAE level in the presence of an external pure tone at $10 \mathrm{~dB} \mathrm{SL}$ at $\mathrm{F}_{x}$. The noise floor (dashed black line) corresponds to about $-14 \mathrm{~dB}$ SPL. Bottom panels: The FDLs measured ipsilaterally (solid black curve) and contralaterally (dashed red curve) to the SOAE in the same subjects are plotted as a proportion of $\mathrm{F}_{x}$. The geometric means and standard deviations over 3 measurement blocks are shown.

\section{DISCUSSION}

The existence of a link between perceptual changes in frequency discrimination and the interaction of SOAEs with external tones was investigated by comparing objective measurements of SOAE entrainment patterns to behavioral FDLs in the same group of subjects. It was found that (1) FDLs in the emission ear were always reduced near the SOAE frequency, (2) this effect was specific to the emission ear, with contralateral FDLs remaining unaffected by the presence of an SOAE in the opposite ear, and (3) the individual variations in FDL around the SOAE frequency were consistent with the subjects' entrainment patterns, with reduced frequency discrimination in the region of strongest beating between the external tone and the SOAE. These findings argue for a peripheral origin, at least in part, for the observed subjective changes in pitch perception around SOAE frequencies, due to the non-linear interaction of SOAEs with external tones in the cochlea. 


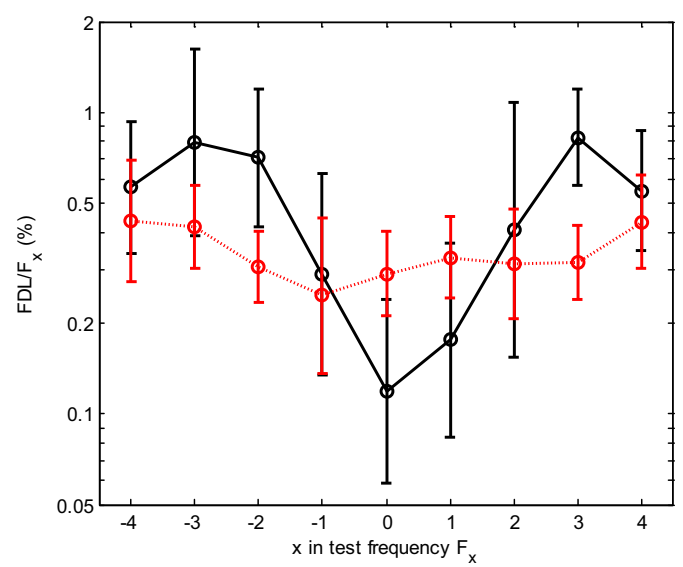

FIGURE 3. Average FDLs measured ipsilaterally (solid black curve) and contralaterally (dashed red curve) to the SOAE at nine test frequencies around the SOAE frequency $\mathrm{F}_{0}$. The means and standard deviations over 6 subjects are shown.

The reduction in ipsilateral FDLs near SOAEs is consistent with the trend observed by Norena et al. (2002), and in conflict with the reports of Köhler and Fritze (1994) that monaural and binaural frequency discrimination, measured at $60 \mathrm{~dB}(\mathrm{~A})$ and $\pm 30 \mathrm{~Hz}$ around the $\mathrm{SOAE}$, is poorer near the emission frequency. A direct comparison with the latter results is difficult since the authors did not show the data, and their experiments were performed at higher levels than in the present study. However, if the pitch of pure tones in the close vicinity to SOAEs is shifted away from the SOAE, as shown by Köhler and Fritze (1994), one could actually expect an improvement in frequency discrimination at $\mathrm{F}_{0}$, due to the enhancement of the pitch separation between tones on each side of $\mathrm{F}_{0}$. Such an improvement was observed by Norena et al. (2002) and confirmed by the present results. Although the present FDLs were generally higher than those obtained by Norena et al. (2002), this was expected due to the difference in presentation level between the two studies (30 vs $10 \mathrm{~dB}$ SL). FDLs are indeed known to improve with level (Wier et al., 1977) and the measured FDLs are in good agreement with the data of Wier et al. for $10 \mathrm{~dB}$ SL.

The absence of a dependence of the contralateral FDLs on $\mathrm{F}_{x}$ in the present study contrasts with the results of Norena et al. (2002) and does not confirm their hypothesis of a central effect of neural reorganization due to the presence of an SOAE. The use of a higher SL in their study, and of the same spacing between test frequencies for all subjects despite widely spread SOAE frequencies, might play a role in this discrepancy. First, a much broader entrainment region would be expected at higher SLs (e.g., Long and Tubis, 1988; Long, 1998), such that the frequency range tested by Norena et al. (2002) might not have been wide enough to observe elevated FDLs due to beating between the SOAE and the external tones. Furthermore, the perceptual effect of beating should be weaker for higher SLs, as the beating amplitude is inversely proportional to the difference in level between the two tones. Therefore, if the perceived beating affects frequency discrimination, this should have a more adverse effect at lower SLs. These last two aspects are consistent with the present findings of much higher FDLs in regions of strong beating $(\approx 1 \%)$ than the most elevated FDLs obtained by Norena et al. $(\approx 0.3 \%)$. The same authors also argued that contralateral presentation of a continuous pure tone at $30 \mathrm{~dB}$ SL produced a similar effect on FDLs to that of an ipsilateral SOAE. However, these observed trends were not supported by statistical significance. Further investigation is thus needed to determine whether lower FDLs can be obtained due to the presentation of a contralateral tone. At this stage, the role of central plasticity effects remains uncertain, although it is possible that such central effects only become observable for tone levels higher than $10 \mathrm{~dB}$ SL.

Overall, ipsilateral FDLs were lower than contralateral FDLs in the SOAE entrainment region. If contralateral FDLs are considered as the "baseline" performance in the absence of perturbation from a neighboring SOAE, this corresponds to an actual improvement in frequency discrimination near the SOAE, in addition to the observed worsening at more remote "beating" frequencies. A first possible explanation could be the lower HTs typically observed near SOAEs (e.g., Schloth, 1983; Long and Tubis, 1988), and the absence of detailed HT fine-structure measurements in the present study. However, it was ensured that all tones were presented at similar SLs via HT interpolation, and the lack of correlation between FDLs and HTs does not favor this explanation. Alternatively, the pitch shifts reported by Köhler and Fritze (1994), provided they only occur in the emission ear, may be responsible for this increase in performance.

Despite the clear correspondence observed between individual entrainment regions and frequency-discrimination performance, the frequency and level drifts of SOAEs over time (e.g., Whitehead, 1991), as well as the variations in 
HT across frequency, imposed limitations on how accurately the regions of entrainment and beating could be determined in relation to the tones presented in the FDL experiment. Although care was taken to readjust $\mathrm{F}_{0}$ and $\mathrm{L}_{0}$ after probe insertion and to spectrally verify the presence of entrainment, the individual "U" or "V" trend in FDLs as a function of $\mathrm{F}_{x}$ was not always centered on $\mathrm{F}_{0}$ as intended, thus complicating the interpretation of average results. This is partly due to the fact that the FDL measurements were performed at the same SL for each $\mathrm{F}_{x}$ value, while the corresponding entrainment region was estimated according to $10 \mathrm{~dB}$ SL relative to $\mathrm{F}_{0}$ only, making it likely for the actual entrainment regions for test frequencies away from $\mathrm{F}_{0}$ to be wider than the estimated $\Delta \mathrm{F}_{\mathrm{E}-}$ and $\Delta \mathrm{F}_{\mathrm{E}+} \cdot \mathrm{The}$ choice of measuring at a fixed SL was made here to avoid a possible confound of known FDL variations with SL (Wier et al., 1977). This should be seen as a compromise, as the alternative of using of a fixed level relative to $\mathrm{L}_{0}$ might have made it easier to ensure that each $\mathrm{F}_{x}$ value fell into the desired region, but would have made the FDL measurement subject to changes in $\mathrm{SL}$ with $\mathrm{F}_{x}$.

Finally, it was assumed here that the sensation of roughness induced by the beating between the SOAE and the external tone was responsible for elevated FDLs. Although all subjects reported that the tonal quality of the stimuli differed between trials, consistent with the "cricket-like" nature of pure tones interacting with an SOAE in the beating region (Long and Tubis, 1988), these subjective changes in tonality were not tracked individually and in a systematic manner as a function of the frequency and level of the external tone. A more thorough investigation of the relationship between these changes in tonal quality and frequency-discrimination performance at different SLs, and of the pitch shifts induced by SOAEs, may clarify the validity of this assumption, and shed light on the perceptual consequences of SOAEs at low vs moderate levels.

\section{ACKNOWLEDGMENTS}

The authors would like to thank Torsten Dau and Bastian Epp for scientific discussions and Michal Fereczkowski for providing his implementation of the hearing-threshold procedure.

\section{REFERENCES}

Gold, T. (1948). "Hearing. II. The physical basis of the action of the cochlea," Proc. R. Soc. Lond. B 135, 492-498.

Kemp, D. T. (1978). "Stimulated acoustic emissions from within the human auditory system,” J. Acoust. Soc. Am. 64, 13861391.

Kemp, D. T. (2008). "Otoacoustic emissions: Concepts and origins," in Active processes and otoacoustic emissions in hearing. Springer Handbook of Auditory Research (Eds. Manley G. A., Fay R. R., Popper, A. N.) Vol. 30, Chapter 1. New York: Springer, pp. 1-38.

Köhler, W., and Fritze, W. (1994). "Pitch is influenced by spontaneous otoacoustic emissions," Acta Otolaryngol. 114, 110-112.

Lecluyse, W., and Meddis, R. (2009). "A simple single-interval adaptive procedure for estimating thresholds in normal and impaired listeners," J. Acoust. Soc. Am. 126, 2570-2579.

Levitt, H. (1971). "Transformed up-down methods in psychoacoustics," J. Acoust. Soc. Am. 49, 467-477.

Long, G. (1998). "Perceptual consequences of the interactions between spontaneous otoacoustic emissions and external tones," Hear. Res. 119, 49-60.

Long, G. R., and Tubis, A. (1988). "Investigations into the nature of the association between threshold microstructure and otoacoustic emissions," Hear. Res. 36, 125-138.

Norena, A., Micheyl, C., Durrant, J. D., Chéry-Croze, S., and Collet, L. (2002). "Perceptual correlates of neural plasticity related to spontaneous otoacoustic emissions?" Hear. Res. 171, 66-71.

Probst, R., Lonsbury-Martin, B. L., and Martin, G. K. (1991). "A review of otoacoustic emissions,” J. Acoust. Soc. Am. 89, 2027-2067.

Schloth, E. (1983). "Relation between spectral composition of spontaneous otoacoustic emissions and fine-structure of threshold in quiet," Acustica 53, 250-256.

Talmadge, C. L., Long, G. R., Murphy, W. J., and Tubis, A. (1993). "New off-line method for detecting spontaneous otoacoustic emissions in human subjects," Hear. Res. 71, 170-182.

Whitehead, M. L. (1991). "Slow variations of the amplitude and frequency of spontaneous otoacoustic emissions," Hear. Res. 53, 269-280.

Wier, C. C., Jesteadt, W., and Green, D. M. (1977). "Frequency discrimination as a function of frequency and sensation level," J. Acoust. Soc. Am. 61, 178-184.

Zurek, P. M. (1981). "Spontaneous narrowband acoustic signals emitted by human ears,” J. Acoust. Soc. Am. 69, 514-523. 